\title{
RESULTADOS DE BIOPSIA GUIADA POR TOMOGRAFÍA AXIAL COMPUTARIZADA EN EL SÍNDROME DE DESTRUCCIÓN VERTEBRAL, EVALUADOS EN DOS INSTITUCIONES DISTINTAS
}

\author{
RESULTADOS DE BIÓPSIA GUIADA POR TOMOGRAFIA COMPUTADORIZADA AXIAL NA SÍNDROME \\ DE DESTRUIÇÃO VERTEBRAL, AVALIADOS EM DUAS INSTITUIÇÕES DISTINTAS
}

\section{RESULTS OF COMPUTERIZED AXIAL TOMOGRAPHY-GUIDED BIOPSY IN THE VERTEBRAL DESTRUCTION SYNDROME, EVALUATED AT TWO DIFFERENT INSTITUTIONS}

Barón Zarate-Kalfopulos ${ }^{1}$, José Ernesto García-Valerio², Guadalupe Sánchez-Bringas' ${ }^{1}$, Luis Miguel Rosales-Olivares' ${ }^{1}$, Armando Alpizar-Aguirre ${ }^{1}$, Alejandro Reyes-Sánchez ${ }^{1}$

\begin{abstract}
RESUMEN
Objetivo: Comparar resultados de biopsias vertebrales guiadas por tomografía axial computarizada, reportados por dos servicios de Anatomía Patológica distintos en pacientes con síndrome de destrucción vertebral para confirmar confiabilidad y utilidad en el diagnóstico. Métodos: Se obtuvieron muestras de tejido de 21 pacientes en el periodo comprendido del 1 de marzo al 15 de julio del 2011 con el diagnóstico del síndrome de destrucción vertebral y a quienes se les realizó biopsia guiada por tomografía axial computarizada. Las muestras se enviaron en forma cegada a dos servicios de patología distintos. Los resultados fueron analizados con el método de comparación de dos proporciones. Resultados: 14 pacientes fueron del género masculino (67\%) y 7 del femenino (23\%), edades de 28-82, con afección principalmente lumbar (48\%) y torácica (38\%); afectados en una vértebra en $62 \%$, en dos en un $33 \%$ y en 3 o más niveles en $5 \%$. Las vértebras más afectadas fueron L1, L2 y L3 (12/30 [40\%]), T4 (3/30 [10\%]) y T9 (3/30 [10\%]). Los resultados se agruparon en categorías: 1. Osteomielitis (9/21 [43\%]), 2. Tumores (7/21 [33\%]), 3. Metástasis (3/21 [14\%]), 4. Tejido normal (2/21 [10\%]), 5. Inflamación (0/21 [0\%]), 6. Muestra inadecuada (0/21 [0\%]), 7. Mal de Pott (0/21 [0\%]). Conclusiones: De acuerdo a los valores de Z obtenidos mediante la prueba de comparación de dos proporciones no se encontró diferencia significativa entre los resultados reportados por dos los dos servicios de patología en el síndrome estudiado, determinándose que esta parte del proceso es confiable y útil en un $90 \%$.
\end{abstract}

Descriptores: Columna vertebral; Síndrome; Biopsia; Biopsia guiada por imagen.

\section{RESUMO}

Objetivo: Comparar resultados de biópsias vertebrais guiadas por tomografia axial computadorizada, relatados por dois serviços de Anatomia Patológica distintos em pacientes com síndrome de destruição vertebral para confirmar a confiabilidade e a utilidade no diagnóstico. Métodos: Foram obtidas amostras de tecido de 21 pacientes no período de 1 de março a 15 de julho de 2011, com diagnóstico de síndrome de destruição vertebral, que foram submetidos a biópsia guiada por tomografia axial computadorizada. As amostras foram enviadas de modo cego a dois serviços de patologia distintos. Os resultados foram analisados pelo método de comparação de duas proporções. Resultados: 14 pacientes eram do sexo masculino (67\%) e 7 do feminino (23\%), com idades entre 28 e 82 e afecção predominantemente lombar (48\%) e torácica (38\%); 62\% tinham comprometimento em uma vértebra, 33\% em duas e $5 \%$ em três ou mais níveis. As vértebras mais afetadas foram L1, L2 e L3 (12/30 [40\%]), T4 (3/30 [10\%]) e T9 (3/30 [10\%]). Os resultados foram agrupados em categorias: 1. Osteomielite (9/21 [43\%]), 2. Tumores (7/21 [33\%]), 3. Metástases (3/21 [14\%]), 4. Tecido normal (2/21 [10\%]), 5. Inflamação (0/21 [0\%]), 6. Amostra inadequada (0/21 [0\%]), 7. Mal de Pott (0/21 [0\%]). Conclusões: De acordo com os valores de Z obtidos pela prova de comparação de duas proporções, não se encontrou diferença significante entre os resultados relatados pelos dois serviços de patologia na síndrome estudada, ficando determinado que essa parte do processo tem confiabilidade e utilidade de $90 \%$.

Descritores: Coluna vertebral; Síndrome; Biópsia; Biópsia guiada por imagem.

\begin{abstract}
Objective: To compare results of vertebral biopsy guided by computerized axial tomography scan, reported by two different Departments of Pathology in patients with vertebral destruction syndrome to confirm the reliability and utility in the diagnosis. Methods: Tissue samples from 21 patients were obtained March 1 to July 15, 2011, with a diagnosis of the vertebral destruction syndrome and who underwent computerized axial tomography-guided biopsy. The samples were blindly sent to two different pathology senvices. The results were analyzed using the two-proportion z-test. Results: 14 patients were male (67\%) and 7 females (23\%), aged between 28 and 82, with condition predominantly lumbar (48\%) and thoracic (38\%); $62 \%$ had one vertebra affected, $33 \%$ ha two and $5 \%$ had three or more levels involved. The most affected vertebrae were $L 1$, $L 2$ and L3 (12/30 [40\%]) and T9 (3/30 [10\%]). The results were grouped into categories: 1. Osteomyelitis (9/21 [43\%]), 2. Tumors (7/21 [33\%]), 3. Metastases (3/21 [14\%]), 4. Normal tissue (2/21 [10\%]), 5. Inflammation (0/21 [0\%]), 6. Inadequate sample (0/21 [0\%]), 7 . Pott's disease (0/21 [0\%]). Conclusions: According to the values obtained by the two-proportion z-test, there was no significant difference between the results reported by the two departments of pathology for the syndrome studied, being determined that this part of the process has reliability and usefulness of $90 \%$.
\end{abstract}

Keywords: Spine; Syndrome; Biopsy; Image-guided biopsy.

1. Servicio de Cirugía de Columna, Instituto Nacional de rehabilitación (INR), Col. Arenal de Guadalupe Delegación Tlalpan, México.

2. Curso de Alta Especialidad en Cirugía de Columna Vertebral da UNAM, México, DF.

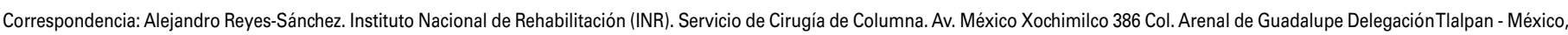
DF. CP 14389. Email: alereyes@inr.gob.mx.

Recibido em 25/04/2013, aceptado em 08/05/2013 


\section{INTRODUCCION}

El Síndrome de Destrucción Vertebral (SDV) se define como una patología de etiología múltiple, caracterizada por alteraciones en la estructura y ultra estructura de la columna vertebral causando deformidad, aumento de volumen circunvecino en uno o más cuerpos vertebrales, que se acompaña de dolor e incapacidad funcional por alteraciones mecánicas y neurológicas. ${ }^{1}$

Sus causas pueden ser: Infección (incluso Tuberculosis), Tumores Primarios, Metástasis, y/o Alteraciones Metabólicas. ${ }^{1}$

Para llegar a un diagnóstico etiológico adecuado se consideran como pruebas de alta sensibilidad a la Tomografía Axial Computarizada (TAC), la Resonancia Magnética, la Gammagrafía, la reacción en cadena de la polimerasa, la velocidad de sedimentación globular, la proteína $\mathrm{C}$ reactiva y la determinación de fosfatasa alcalina y ácida. Otras pruebas tienen baja sensibilidad y especificidad diagnóstica. 2,3 Existen dos tipos fundamentales de biopsia ${ }^{4}$ : a) abierta $^{3}$, b) percutánea o por punción. Para su realización se requiere de apoyo radiológico (Fluoroscopio, Ultrasonido o TAC). Existen factores, que habrán de considerarse cuando se realiza una biopsia vertebral guiada con $\mathrm{TAC}^{3}$ : a) humano, b) imagenlogía previa para su planeación, c) características de la lesión, d) la preparación del paciente, e) posición del paciente, f) el sitio de obtención, g) topografía de la lesión, h) tamaño de las muestras, i) tipo de aguja con la cual se realiza la biopsia, j) si la biopsia es única o múltiple (77\% vs 94\% de exactitud diagnóstica). La obtención de una muestra inadecuada o insuficiente constituye el mayor problema de la biopsia guiada por TAC. . $^{5}$

La biopsia vertebral percutánea fue descrita por primera vez en 1935 por Robertson y Ball. ${ }^{10} \mathrm{Y}$ ha sido mejorada y sistematizada por varios autores. ${ }^{11-18}$

El mérito de la utilización de la TAC para la realización de las biopsias vertebrales se atribuye a Adapon et al. ${ }^{19}$ en 1981, quien demostró el valor de dicha técnica y cuya aportación sirvió para que se desarrollara hasta que en la actualidad persiste con tecnología superior tanto en el equipo de radiodiagnóstico como en las agujas y trocares. ${ }^{20-25}$

En 2007 en nuestro centro, se evaluó la utilidad de la biopsia transpedicular percutánea guiadas con un intensificador de imágenes en brazo en $\mathrm{C}$, en el diagnóstico definitivo en los pacientes con destrucción vertebral, con resultados poco alentadores ya que se concluyó que la biopsia sola, como elemento diagnóstico fue útil sólo en $55 \%$ de los casos, contrastando con la literatura mundial. ${ }^{1}$ Por lo anterior se optó por implementar la biopsia guiada por TAC, se elaboraron guías diagnósticas para disminuir la probabilidad de obtener muestras insuficientes o inadecuadas al momento de obtener la biopsia basándonos, en publicaciones previas. ${ }^{1,3}$

De esta forma el objetivo del estudio fue comparar los resultados reportados por dos servicios de patología distintos de la biopsia vertebral guiada por Tomografía Axial Computarizada en el Síndrome de Destrucción Vertebral. Nuestra Hipótesis fue que los resultados histopatológicos de las biopsias vertebrales dirigidas por Tomografía Axial Computarizada en el Síndrome de Destrucción Vertebral, valorados en dos servicios de patología distintos, no tendrían diferencias.

\section{MATERIAL Y METODOS}

Se realizó un estudio transversal en el cual se incluyeron pacientes consecutivos de cualquier edad o género, que fueron hospitalizados de forma en el servicio con diagnóstico radiológico de Síndrome de Destrucción Vertebral, en el periodo comprendido del 1 de marzo al 15 de Julio del 2011; que no tuvieran al momento de la hospitalización diagnóstico etiológico y que bajo consentimiento informado, se les hubiera realizado biopsia guiada por TAC y enviada para su análisis al patólogo de nuestro centro y a otro patólogo externo.

Se excluyeron las biopsias de pacientes, que fueron obtenidas con otro apoyo radiológico (fluoroscopio, ultrasonido, RMN).

Fueron eliminados del estudio los pacientes a los que se les realizó únicamente biopsia a cielo abierto; aquellos cuya muestra obtenida fue analizada por un solo patólogo y en los cuales ambos patólogos reportaron la muestra como insuficiente o no adecuada para el diagnóstico. La biopsia se realizó por médicos especialistas quienes se encuentran cursando el posgrado en Cirugía de Columna en el Instituto Nacional de Rehabilitación. Para guiar el procedimiento se utilizó un Tomógrafo con rápida adquisición de imágenes y reconstrucción multiplanar (Light Speed VZT, General Electric 2007) el cual fue manejado por el médico y técnico radiólogo del Servicio de Imagenología de nuestro hospital. Para la realización de la biopsia se utilizó una aguja de Jamshidi (Cardinal Health Co.) con un diámetro de $5 \mathrm{~mm}$ y $15 \mathrm{~cm}$ de longitud. La toma de la biopsia se realizó por la vía transpedicular. Durante la inserción de la aguja se obtuvieron controles tomográficos a fin de verificar la adecuada dirección y profundidad y evitar así el daño a estructuras nerviosas. Una vez que se llegó al sitio afectado más representativo de la lesión se retiró la trefina, se aspiró el contenido de la lesión y se retiró la camisa de la aguja ejerciendo simultáneamente presión negativa para obtener la muestra tanto de tejido blando, de material líquido, y de tejido óseo. Al retirar la aguja de Jamshidi se obtuvo control tomográfico del sitio puncionado a fin de corroborar que no existiera sangrado. El resultado sólido obtenido (hueso o tejido blando) se dividió en 4 partes, las partes 1 y 3 se enviaron a un patólogo y las partes 2 y 4 se enviaron al otro patólogo. La muestra líquida se colocó en un medio de transporte de Stuart y se envió al laboratorio de bacteriología para su estudio. Los diagnósticos histopatológicos fueron clasificados en 7 categorías: 1. Osteomielitis, 2. Tumores, 3. Metástasis, 4. Tejido Normal, 5. Inflamación, 6. Muestra inadecuada, 7. Mal de Pott. Estas categorías fueron reportadas por alguno de los patólogos en al menos uno de los resultados de los 21 pacientes estudiados.

\section{Análisis Estadístico}

Se describieron las variables por medio de medidas de frecuencia, tendencia central y dispersión. Los resultados de cada patólogo fueron analizados con la prueba de comparación de dos proporciones, considerando un nivel de significancia de 0.05. Se usó el paquete estadístico Microsoft Excel 2007.

\section{RESULTADOS}

Se analizaron muestras de 21 pacientes. Los resultados muestran que 14/21 pacientes fueron hombres (67\%) y 7/21 fueron mujeres (23\%), con una media de edad de 59 años, el grupo de edad más prevalente fue el de 51 a 60 años. (Tabla 1) La afección de una sola vértebra se presentó en $62 \%$ de los pacientes, el 33\% presentó el daño en dos vértebras y sólo en 5\% hubo afección de 3 o más vértebras. (Tabla 2) El segmento vertebral más afectado fue el lumbar (48\%), seguido del torácico (38\%) (Tabla 3) Las vértebras más afectadas fueron L1, L2 y L3 [12 (40\%) de 30], seguidas de T4 [3 (10\%) de 30] y T9 [3 (10\%) de 30]. (Figura 1) Los resultados histopatológicos definitivos se agruparon en 7 categorías: 1.Osteomielitis [9 (43\%) de 2]), 2. Tumores [7 (33\%) de 21], 3. Metástasis [3 (14\%) de 21], 4. Tejido Normal [2 (10\%) de 2]), 5. Inflamación [0 (0\%) de 21], 6. Muestra inadecuada [0 (0\%) de 21], 7. Mal de Pott [0 (0\%) de 21]. (Tabla 4)

\section{DISCUSION}

Una biopsia bien realizada es aquella en que se obtiene información suficiente para obtener un diagnóstico correcto y establecer un plan adecuado de tratamiento, todo esto sin complicaciones y sin modificar el tratamiento quirúrgico ni la evolución del proceso patológico. ${ }^{5,6}$ La biopsia es imprescindible en aquellas alteraciones cuya la obtención de muestra de tejidos pueda ser determinante para su diagnóstico, estatificación o tratamiento. ${ }^{3}$ Desde hace más de 75 años que Robertson y Ball ${ }^{10}$ describieron por primera vez la técnica para obtención de biopsias vertebrales, sin embargo, no fue sino hasta 1981 cuando Adapon et al. ${ }^{19}$ utiliza la guía tomográfica 
Tabla 1. Características demográficas de los pacientes con síndrome de destrucción vertebral.

\begin{tabular}{c|c|c|c}
\hline Género & & $\mathbf{n = 2 1}$ & $\%$ \\
\hline & Femenino & 7 & 33 \\
\hline Edad & Masculino & 14 & 67 \\
\hline & Mínima & Años & \\
\hline & Máxima & 28 & \\
\hline & Promedio & 82 & \\
\hline & Promedio en hombres & 59 & \\
\hline & Promedio en mujeres & 62.86 & \\
\hline & Rangos & $\mathbf{n = 2 1}$ & $\%$ \\
\hline & 28 a 30 & 1 & 5 \\
\hline & 31 a 40 & 2 & 10 \\
\hline & 41 a 50 & 2 & 10 \\
\hline & 51 a 60 & 7 & 33 \\
\hline & 61 a 70 & 4 & 19 \\
\hline & 71 en adelante & 5 & 24 \\
\hline
\end{tabular}

Tabla 2. Número de vértebras afectadas por paciente.

\begin{tabular}{c|c|c}
\hline Número de vértebras & Número de pacientes $(\mathbf{n = 2 1 )}$ & $\%$ \\
\hline 1 & 13 & 62 \\
\hline 2 & 7 & 33 \\
\hline 3 & 1 & 5 \\
\hline Total & 30 & 100 \\
\hline
\end{tabular}

Tabla 3. Distribución del daño por segmento en la columna vertebral.

\begin{tabular}{c|c|c}
\hline Segmento vertebral & $\mathbf{n = 2 1}$ & $\%$ \\
\hline Cervical & 0 & 0 \\
\hline Torácico & 8 & 38 \\
\hline Lumbar & 10 & 48 \\
\hline Sacro & 1 & 5 \\
\hline Cervical-torácico & 1 & 5 \\
\hline Torácico-lumbar & 1 & 5 \\
\hline
\end{tabular}

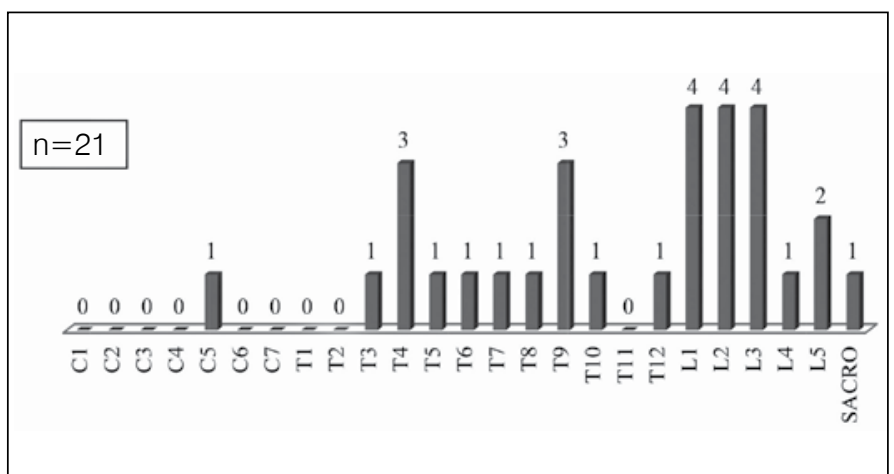

Figura 1. Distribución de las vértebras afectadas en los pacientes con síndrome de destrucción vertebral.
Tabla 4. Análisis comparativo de los resultados histopatológicos obtenidos por dos patólogos distintos.

\begin{tabular}{c|c|c|c|c}
\hline Categoría & $\begin{array}{c}\text { Patólogo } \\
\text { Interno }\end{array}$ & $\begin{array}{c}\text { Patólogo } \\
\text { Externo }\end{array}$ & Valor de Z* & $\begin{array}{c}\text { Diagnóstico } \\
\text { Definitivo** }\end{array}$ \\
\hline Osteomielítis & 8 & 9 & -0.205 & $9(43 \%)$ \\
\hline Tumores & 6 & 3 & 0.909 & $7(33 \%)^{(y)}$ \\
\hline Metástasis & 3 & 3 & 0 & $3(14 \%)^{(y)}$ \\
\hline Tejido normal & 2 & 2 & 0 & $2(10 \%)$ \\
\hline Inflamación & 0 & 3 & -1.677 & 0 \\
\hline Muestra inadecuada & 2 & 1 & 0.558 & 0 \\
\hline Mal de Pott & 0 & 0 & 0 & 0 \\
\hline
\end{tabular}

$\mathrm{n}=21, \mathrm{p}=0.05^{\star}$ El valor crítico de $\mathrm{Z}$ fue de $1.966(+\mathrm{y}-)^{* *}$ *El diagnóstico definitivo se estableció ya sea por: a) Opinión del experto (oncólogo, infectólogo, etc.), b) Cultivo bacteriológico, c) Análisis global del total de estudios complementarios a la biopsia. (y) La suma de estos valores es igual a $47 \%$, y corresponde al porcentaje en que el estudio histopatológico como único estudio es útil para el diagnóstico definitivo.

para la obtención de dicha biopsia estableciendo así un estándar en este procedimiento, ${ }^{3}$ ya que reportó porcentajes de utilidad del $95 \%{ }^{19}$ en el diagnóstico definitivo.

La reproducción estandarizada no ha sido fácil en todo el mundo por la poca accesibilidad en esos años para la obtención de un estudio tomográfico. Pero en 1986 Laredo y Bard ${ }^{18}$ publica tasas de utilidad del $90 \%$ en la biopsia guiada por fluoroscopio, haciendo más accesible la utilización de este recurso para la obtención de la biopsia vertebral. Por tal motivo inicialmente en nuestro centro fue utilizado este recurso; sin embargo no fue posible reproducir la utilidad reportada por Laredo ya que la muestra sólo fue adecuada para el diagnóstico en un 55\%, ${ }^{25}$ tasas consideradas como inadecuadas en nuestro medio para la obtención del mismo.

Con la adquisición de un tomógrafo con rápida adquisición de imágenes y reconstrucción multiplanar (Light Speed VZT, General Electric 2007) en nuestro centro, se abrió la posibilidad de su utilización en la biopsia vertebral en el síndrome de destrucción vertebral, y con ello, de mejorar las tasas de certeza diagnóstica; sin embargo, otra parte de la certeza diagnóstica, la centrada en el resultado histopatológico aún debía ser probada. El estudio se realizó en ciego, aunque cabe señalar que no se valoró la concordancia entre los patólogos debido a que no se cumplieron los requisitos para este tipo de análisis. Si bien es cierto que en este estudio se decidió fragmentar la muestra obtenida para ser enviada a dos patólogos, la fragmentación (en 4 partes: partes 1 y 3 para un patólogo y partes 2 y 4 al otro) no originó valores significativamente altos en el resultado denominado "tejido normal" (10\%), originando una certeza en el diagnóstico del $90 \%$, el cual es comparable con las tasas reportadas en grandes series de casos. ${ }^{3} Y$ si bien es cierto que existió el diagnóstico de "muestra inadecuada" [2 (10\%) de 21 por el patólogo interno y 1(5\%) de 21 por el patólogo externo], no hubo ningún caso en el que este reporte fuera corroborado por el otro patólogo. (Tabla 4)

En este estudio, la biopsia vertebral y el estudio histopatológico solo, fue suficiente en un $47 \%$ para la obtención del diagnóstico definitivo, pues como se puede observar en los resultados, 9 (42\%) de 21 de los resultados obtenidos se reportaron como osteomielitis por el patólogo. Estas muestras requirieron la realización de un estudio bacteriológico adicional para llegar al diagnóstico etiológico.

Por otro lado, llama la atención la similitud del 100\% en los resultados reportados como "metástasis" (Tabla 4) lo cual es comparable con grandes series de casos que indican que la biopsia guiada por tomografía es útil en 95-100\% de los casos. ${ }^{3,21}$

Este estudio tiene el inconveniente de tener una muestra pequeña de pacientes $(n=21)$, sin embargo, es el punto de partida para otros trabajos de investigación con el mismo diseño de estudio.

Una limitación del estudio es que al obtener dos opiniones distintas de un resultado histopatológico, se está obligado a determinar 
cuál de los dos resultados será el definitivo cuando estos no son iguales, para ello se estableció como diagnóstico definitivo: a) la opinión del experto (oncólogo en el caso de malignidad, infectólogo en el caso de infección), b) el cultivo bacteriológico, c) la interpretación global del resto de los estudios establecidos en el protocolo diagnóstico previamente reportado ${ }^{3} \mathrm{o}$ d) el resultado de la biopsia abierta.

Se utilizó la técnica de la comparación entre dos proporciones para determinar la similitud de dos resultados, ya que no era posible utilizar la prueba de concordancia debido a que por razones de conveniencia no se notificó a ninguno de los patólogos involucrados de la existencia de otro patólogo que estuviera analizando otra parte de la muestra obtenida del mismo paciente.

El diagnóstico reportado como "tejido normal" [2 casos (10\%) de 21] en realidad es considerado en este estudio como falla diagnóstica toda vez que ambos patólogos coincidieron en el resultado, lo cual propició que estos pacientes requirieran la realización de biopsia abierta para llegar al resultado definitivo. Este resultado inadecuado puede deberse a: 1) la obtención de tejido en la biopsia en un sitio no representativo, 2) la mala técnica para la obtención o el envío de la muestra y 3) otras causas no atribuibles al patólogo.

\section{CONCLUSIONES}

De acuerdo a los valores de Z obtenidos mediante la prueba de comparación de 2 proporciones no se encontró diferencia significativa en los resultados reportados por los dos patólogos que participaron en este estudio; determinándose que la biopsia guiada por TAC es útil y confiable en un $90 \%$ de los casos en nuestro medio para obtener resultado definitivo en el síndrome de destrucción vertebral.

Todos los autores declaran no existir ningún potencial conflicto de intereses referente a este artículo.

\section{REFERENCIAS}

1. Rosales-Olivares LM, Valle-Cerna I, Alpizar-Aguirre A, Miramontes-Martínez V, ArenasSordo Mde L, Reyes-Sánchez A. Percutaneous biopsy evaluation in the diagnosis of thoracic and lumbar vertebral destruction syndrome. Cir. 2007:75(6):459-63.

2. Alpizar-Aguirre A, Elías-Escobedo A, Rosales-Olivares LM, Miramontes-Martínez V, Reyes-Sánchez A. Vertebral destruction syndrome. Diagnostic evaluation systems. Cir. 2008;76(3):205-11.

3. Rimondi E, Rossi G, Bartalena T, Ciminari R, Alberghini M, Ruggieri P, Errani C, et al. Percutaneous CT-guided biopsy of the musculoskeletal system: results of 2027 cases. Eur J Radiol. 2011;77(1):34-42

4. Stoker DJ. Bone Biopsy. In: Resnick D, Petterson H editors. Skeletal Radiology. London: Merit Communications; 1992. p. 81-92.

5. Barth RJ Jr, Merino MJ, Solomon D, Yang JC, Baker AR. A prospective study of the value of core needle biopsy and fine needle aspiration in the diagnosis of soft tissue masses. Surgery. 1992;112(3):536-43.

6. Springfield DS, Rosenberg A. Biopsy: complicated and risky. J Bone Joint Surg Am. 1996:78(5):639-43.

7. Jelinek JS, Murphey MD, Welker JA, Henshaw RM, Kransdorf MJ, Shmookler BM, et al Diagnosis of primary bone tumors with image-guided percutaneous biopsy: experience with 110 tumors. Radiology. 2002;223(3):731-7.

8. Dupuy DE, Rosenberg AE, PunyaratabandhuT, Tan MH, Mankin HJ. Accuracy of CT-guided needle biopsy of musculoskeletal neoplasms. AJR Am J Roentgenol. 1998;171(3):759-62.

9. Hau A, Kim I, Kattapuram S, Hornicek FJ, Rosenberg AE, Gebhardt MC, et al. Accuracy of CT guided biopsies in 359 patients with musculoskeletal lesions. Skeletal Radiol. 2002:31(6):349-53.

10. Robertson RC, Ball RP. Destructive spinal lesions: Diagnosis by needle biopsy. J Bone Joint Surg. 1935;17:749-58.

11. Valls J, Ottolenghi CE, Schajowicz F. Aspiration biopsy in diagnosis of lesions of vertebral bodies. J Am Med Assoc. 1948;136(6):376-82

12. Ray RD. Needle biopsy of the lumbar vertebral bodies; a modification of the Valls technique. J Bone Joint Surg Am. 1953;35(3):760-2
13. Frankel CJ. Aspiration biopsy of the spine. J Bone Joint Surg Am. 1954;36(1):69-74.

14. Ottolenghi CE. Diagnosis of orthopaedic lesions by aspiration biopsy; results of 1,061 punctures. J Bone Joint Surg Am. 1955;37-A(3):443-64

15. Craig FS. Vertebral-body biopsy. J Bone Joint Surg Am. 1956;38(1):93-102.

16. Ackermann W. Vertebral trephine biopsy. Ann Surg. 1956;143(3):373-85.

17. Ottolenghi CE. Aspiration biopsy of the spine. Technique for the thoracic spine and results of twenty-eight biopsies in this region and over-all results of 1050 biopsies of other spinal segments. J Bone Joint Surg Am. 1969;51(8):1531-44.

18. Larédo JD, Bard M. Thoracic spine: percutaneous trephine biopsy. Radiology. 1986;160(2):485-9.

19. Adapon BD, Legada BD Jr, Lim EV, Silao JV Jr, Dalmacio-Cruz A. CT-guided closed biopsy of the spine. J Comput Assist Tomogr. 1981;5(1):73-8.

20. Kattapuram SV, Rosenthal DI. Percutaneous biopsy of the cervical spine using CT guidance. AJR Am J Roentgenol. 1987;149(3):539-41.

21. Lis $E$, Bilsky MH, Pisinski L, Boland P, Healey JH, O'malley $B$, et al. Percutaneous CTguided biopsy of osseous lesion of the spine in patients with known or suspected malignancy. AJNR Am J Neuroradiol. 2004;25(9):1583-8.

22. Ashizawa R, Ohtsuka K, Kamimura M, Ebara S, Takaoka K. Percutaneous transpedicular biopsy of thoracic and lumbar vertebrae--method and diagnostic validity. Surg Neurol. 1999;52(6):545-51

23. Stringham DR, Hadjipavlou A, Dzioba RB, Lander P. Percutaneous transpedicular biopsy of the spine. Spine (Phila Pa 1976). 1994;19(17):1985-91.

24. Sucu HK, Bezircioglu H, Ciçek C, Erşahin Y. Computerized tomography-guided percutaneous transforaminodiscal biopsy sampling of vertebral body lesions. J Neurosurg. 2003;99(Suppl 1):51-5.

25. Sucu HK, Ciçek C, Rezanko T, Bezircioğlu H, Erşahin Y, Tunakan M, et al. Percutaneous computed-tomography-guided biopsy of the spine: 229 procedures. Joint Bone Spine. 2006;73(5):532-7. 